\title{
Los clásicos como resistencia: la lectura literaria en el marco de una educación lectora transmedia ${ }^{\star}$
}

\section{The Classics as Resistance: literary reading within the framework of a transmedia reading education}

\author{
Josep Ballester Roca \\ Universitat de València \\ josep.ballester@uv.es \\ ORCID ID: https://orcid.org/0000-0002-1995-3253
}

\section{Jeroni Méndez Cabrera}

Universitat de València

\section{jeronimo.mendez@uv.es}

ORCID ID: https://orcid.org/0000-0003-2248-6577

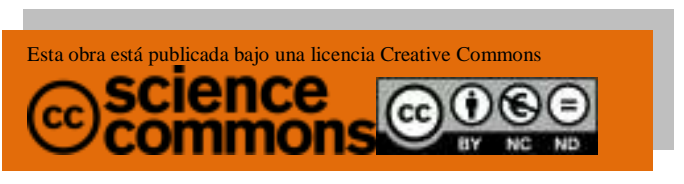

DOI: $10.17398 / 1988-8430.34 .195$

Fecha de recepción: 10/03/2021 Fecha de aceptación: 24/05/2021

Ballester Roca, J., y Méndez Cabrera, J. (2021). Los clásicos como resistencia: la lectura literaria en el marco de una educación lectora transmedia. Tejuelo, 34, 195-220.

Doi: https://doi.org/10.17398/1988-8430.34.195

$*$

Este trabajo se ha realizado en el marco del Proyecto de Investigación de Investigación I+D "YOUTUBERS E INSTAGRAMMERS: LA COMPETENCIA MEDIÁTICA EN LOS PROSUMIDORES EMERGENTESRTI2 018-093303-BI00. Ministerio de Economía y Competitividad. Además también se ha realizado en el marco del proyecto de innovación "Educación literaria, alfabetitzación multimodal y canon" de la Universitat de València (UV-SFPIE_PID19-1098237). 
Resumen: Actualmente, existe una propagación de títulos narrativos inspirados por estructuras argumentales, personajes, temas y arquetipos procedentes de diversos clásicos literarios, historias que trascienden las páginas de los libros y llegan a las grandes pantallas de cine, a las pequeñas de los dispositivos móviles o a plataformas diversas de entretenimiento interactivo: diversos servicios de streaming e incluso videojuegos. La lectura literaria hoy en día implica la expansión de la cultura escrita y los referentes literarios a través de modos múltiples y medios de expresión mediante formas de interpretación que integran diferentes tipos de discursos (textuales, visuales, sonoros, interactivos) pero que resultan a la vez convergentes en cuanto a los significados culturales. Concibiendo las nuevas alfabetizaciones lectoras como experiencias mediáticas integradas, que incluyen propuestas y proyectos transmedia para la educación literaria, como estudio de caso significativo, en este trabajo se procederá al análisis cualitativo de Red Dead Redemption 2 como muestra paradigmática de videojuego que presenta interesantes elementos intradiegéticos e intertextualidades favorables al fomento lector, considerando sus implicaciones en lo referente a la lectura de ciertos clásicos literarios y valorando las posibilidades de este medio para el desarrollo de la competencia literaria.
Abstract: Nowadays, there is a spread of narrative titles inspired by plot structures, characters, themes and archetypes from various literary classics, stories that transcend the pages of books and reach the big cinema screens, the small screens of mobile devices or some platforms of interactive entertainment: streaming services and even video games. Literary reading at present implies the expansion of written culture and literary references through multiple media and modes of expression through forms of interpretation that integrate different types of discourses (textual, visual, sound, interactive) but that result converging on cultural meanings at the same time. Conceiving the new reading literacies as integrated media experiences, which include transmedia proposals and projects for literary education, as a significant case study, in this work we will proceed to the qualitative analysis of Red Dead Redemption 2 as a paradigmatic sample of a video game that presents interesting intradiegetic elements and intertextualities that positively stimulate reading promotion, considering its implications in relation to the reading of certain literary classics and assessing the possibilities of this medium for the development of literary competence.

Keywords: classics; literature; reading; videogames; education.

Palabras clave: clásicos; literatura; lectura; videojuegos, educación. 


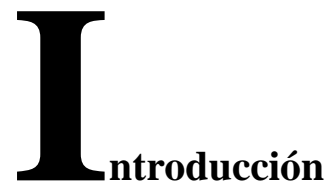

En la actualidad, existe una proliferación de títulos narrativos inspirados por estructuras argumentales, personajes y temas originarios de diversos clásicos literarios y sugieren procesos diversos de adaptación o al menos de intertextualidad; se trata de propuestas narrativas que van más allá de las páginas de los libros y llegan a las grandes pantallas de cine o las pequeñas de los dispositivos móviles, a las televisiones inteligentes $\mathrm{o}$, incluso, a plataformas diversas de entretenimiento interactivo: servicios de streaming, álbumes digitales, e incluso videojuegos. La lectura literaria hoy en día implica la expansión de la cultura escrita, las narrativas y los referentes literarios a través de modos múltiples y medios de expresión exigiendo un proceso de lectura que incluye diferentes tipos de discursos (textuales, visuales, sonoros, interactivos) pero que resulta a la vez convergente en cuanto a los significados culturales (Jenkins, 2006; Scolari, 2013, 2018; Baker, 2013; Cope y Kalantzis, 2009, 2015). Es lo que en el marco de la formación lectora y literaria, algunos autores denominan, hablando de nuevas alfabetizaciones lectoras, experiencia mediática integrada (López, Jerez y Encabo, 2015); una forma de lectura que, en virtud de esa expansión narrativa en diversos medios y soportes, requiere de ciertas propuestas y proyectos transmedia para la educación literaria (Hernández Ortega y Rovira-Collado, 2020; Martínez Urbano, 2021). 
Ahora bien, a pesar de este numeroso abanico de opciones para la recepción de textos multimodales y ficciones audiovisuales, la potencialidad fruitiva de los clásicos —independientemente del texto, género o soporte - solo llega a hacerse realmente efectiva en lectores maduros, con una historia lectora amplia, un intertexto lector rico, y una competencia literaria desarrollada (Mendoza, 2001; Herrera y Saavedra, 2012). Sin embargo, para conseguir esto, se tiene que iniciar el camino en la etapa de formación, en la que el docente, en su papel de mediador - gracias al conocimiento de la historia literaria y el dominio de los instrumentos de análisis y valoración de las obras, ya sean textos originales, adaptaciones o versiones-, ejerza una serie de funciones educativas que posibiliten el perfeccionamiento del gusto por la literatura y la consolidación de hábitos de lectura (Sotomayor, 2013; Méndez, 2017; Ballester-Roca e Ibarra-Rius, 2019).

Así pues, ¿es posible fomentar la lectura literaria en pleno siglo XXI teniendo en cuenta las ventajas y limitaciones que ofrece un medio como el de los videojuegos? ¿Se podría, desde la investigación en didáctica de la literatura como educación lectora y literaria, abrazar esta perspectiva para el conocimiento de los clásicos? ¿Existe alguna alternativa, en cualquier caso, que no sea considerar las posibilidades existentes para la animación a la lectura ante las prácticas letradas y el ocio digital de los más jóvenes? Senís y Cerrillo (2005) planteaban ya una reflexión en este sentido hace más de una década:

Internet, los ordenadores, los vídeo-juegos forman parte de su paisaje vital, de su cotidianidad. En virtud de este contexto de recepción, la lectura y la relación de los niños con los libros pueden cambiar. Hace falta, por tanto, considerar esta situación e intentar proponer conceptos en torno al niño lector y a su relación con la literatura -también a su educación literaria- que sean adecuados para explicar y describir este nuevo estado de cosas (p. 65).

La consideración de los clásicos literarios como obras imperecederas que conectan con el lector a través del tiempo y del espacio, que han marcado una época y continúan vivos en el imaginario colectivo, hace que nos planteemos su papel en un momento en el que, precisamente, el imaginario colectivo surge no tanto de los libros, las 
tradiciones orales o el folklore como cultura inmaterial como del uso de las nuevas tecnologías, en un proceso de apropiación de referentes que podemos adjetivar de transcultural y transmedia, tal y como definió este concepto Scolari (2013). Por lo tanto, a razón de esto, hará falta que los clásicos aparezcan, en el momento oportuno y de la forma más propicia, en el itinerario de experiencias lectoras de niños y adolescentes, con el objetivo de proporcionar las bases de una educación literaria significativa que entienda la tradición literaria más allá de la perspectiva historicista o meramente libresca y considere las nuevas formas de lectura y las tecnologías interactivas. Ordine (2017) hablaba de la importancia cultural y la necesidad de recuperar el honor de los clásicos como referentes para entender la vida, más allá de las imposiciones del mercado, de las nuevas tecnologías e incluso más de allá de la didáctica. El caso es que, en la vida de niños, adolescentes y estudiantes universitarios, a día de hoy, también tienen cada vez mayor presencia los videojuegos; bien como mero pasatiempo, como producto de ocio en el tiempo que nos queda libre después de las restricciones de la pandemia global, o incluso como experiencia cultural.

En este sentido, desde la educación literaria se tienen que considerar determinados criterios didácticos y técnicas de mediación que permiten el acceso a la tradición literaria teniendo en cuenta también las ficciones multimodales. La educación literaria, en este contexto, tiene que asegurar la posibilidad de las relaciones intertextuales significativas por parte de los lectores, el conocimiento de los clásicos como lecturas que son la base de mundos de ficción contemporáneos a los que se accede a través de la pantalla. El enriquecimiento del intertexto lector es, por lo tanto, una de las consecuencias del desarrollo de la competencia literaria a través de las opciones de la multimodalidad. La capacidad de relacionar las narrativas actuales, en cualquier género y soporte (en expansiones transmediáticas o no), con la lectura de los clásicos literarios es, de hecho, la evidencia de una competencia literaria desarrollada.

La lectura literaria puede ser previa, simultánea o posterior a la recepción de estas ficciones, con las cuales mantiene unos determinados vínculos a través de los temas, tópicos, motivos o arquetipos 
compartidos. El lector competente en el siglo XXI reencuentra (relee), pues, los clásicos en las películas, las series, los cómics o los videojuegos, por un lado; y de la otra, ha de saber acceder a la tradición literaria a partir de obras no estrictamente literarias. Porque, de hecho, es muy probable que se encuentre antes con la versión cinematográfica, el videojuego correspondiente o la adaptación en serie de televisión, por ejemplo, que con la obra original (sobre todo, si todavía no ha logrado un nivel suficiente de competencia lectora).

En cualquier caso, en el lector competente, el hallazgo de la coincidencia entre textos será motivo de placer estético, de goce intelectual o de satisfacción emocional. Las propuestas de educación lectora y literaria en el siglo XXI han de garantizar justamente esto. Precisamente, como estudio de caso significativo, en este trabajo se procederá al análisis descriptivo de Red Dead Redemption 2 (Rockstar, 2018), como muestra paradigmática de videojuego que presenta sugerentes elementos intradiegéticos e intertextualidades que consideramos favorables al fomento lector, valorando sus implicaciones en lo referente a la lectura de ciertos clásicos literarios y estimando las posibilidades de este medio en el marco educativo.

\section{Marco teórico: videojuegos y educación literaria}

Partimos de la idea de fomento lector como el conjunto de actividades, técnicas y estrategias que persiguen la creación y fomento de hábitos lectores sólidos y estables, con la finalidad de formar lectores activos, críticos y competentes, capaces de comprender textos literarios diversos y de relacionar una historia con su contexto, de una manera lúdica, creativa y placentera, en diferentes momentos respecto al acto de lectura, para la promoción de experiencias lectoras orientadas por el docente. En este sentido, se trata de una concepción convencional de animación a la lectura entendida como todas las formas posibles de promover la lectura literaria desde instancias educativas, que pretende el desarrollo competencial del lector como objetivo último (Colomer, 1995; Cerrillo y García Padrino, 1996; Mendoza, 2001; Ballester, 2015). 
A partir de este concepto, resulta necesaria la integración de diferentes medios y la consideración de las experiencias de lectura transmedia, favoreciendo así las posibilidades de la educación lectora a través del universo de mundos de ficción que la realidad multimedia nos ofrece en la actualidad. Consideramos, además, el papel de los clásicos literarios en todo ello como hipotextos que nutren muchas de las nuevas propuestas de lectura y entretenimiento digital. Por tanto, desde la formación lectora y literaria cabe la opción de aprovechar esta circunstancia para la reivindicación de la lectura de los clásicos partiendo, si fuera necesario, de la experiencia de juego, del game play, como experiencia estética y cultural. En esta línea de actuación pedagógica y de investigación, cualquier trazo de intertextualidad resultará, así, de utilidad fruitiva y didáctica para el profesor de lengua y literatura.

El concepto de narrativas transmedia es imprescindible para conocer los múltiples soportes a través de los que podemos comprender e interpretar una narración (Scolari, 2018; Rovira-Collado, 2019; Chun, 2020). Aunque originariamente el concepto transmedia implica el desarrollo de un argumento a través de distintos medios (libros, películas, cómics, videojuegos, redes sociales), hemos decido utilizar este término para definir la pluralidad de soportes (de caminos multimodales) a través de los que podemos acceder al conocimiento de los clásicos literarios desde la perspectiva de la educación lectora. Analizar, entender y valorar, desde este punto de vista, las nuevas propuestas lúdicas digitales, supone un requisito para la tarea de recuperación de los clásicos literarios como propuesta capaz de aprovechar cualquier sustentáculo mediático para el fomento lector, lo que denota cierta persistencia en el acceso a las tradiciones literarias en la sociedad digital en la que vivimos. Así pues, aunque los trabajos precedentes destinados al estudio de las aportaciones de los videojuegos en la educación literaria ya apuntaban la existencia de referencias a obras de la literatura universal y la inclusión de referencias intertextuales, además de diversas ventajas en lo referente al desarrollo de la competencia lectora (Gee, 2003; Ramírez, 2012; Castro, 2015; Torres-Toukoumidis, Romero-Rodríguez, Pérez-Rodríguez, y Björk, 
2016; Serna y Rovira-Collado, 2016; Serna-Rodrigo, 2020, Gutiérrez Ángel, 2020), cabe destacar la necesidad de estudios específicos donde se analicen desde esta perspectiva las posibilidades concretas de videojuegos para la praxis educativa.

\section{Metodología: la experiencia de juego como propuesta de lectura analítica}

Teniendo en cuenta un acercamiento cualitativo al objeto de estudio, basado en los preceptos de la estética de la recepción (Mayoral, 1987) y los fundamentos constructivistas de la educación lectora y literaria (Mendoza, 2004; Colomer, 2010; Ballester, 2015), alejándonos conscientemente los postulados positivistas en las ciencias sociales, para contribuir a una teoría crítica de la educación y avanzar en un modelo de aprovechamiento didáctico de las narrativas digitales como expansión de la cultura escrita, nuestro trabajo ofrece un estudio descriptivo centrado en lo que podemos considerar una lectura literaria del título comercial Read Dead Redemption 2 (RDR2); basándonos para ello en un proceso analítico de más de 450 horas de experiencia de juego (97\% de compleción del juego, 100\% en el denominado modo historia o narrativo).

Ello nos ha permitido establecer algunas categorías de análisis vinculadas a elementos que permiten el fomento lector entendido como proceso de mediación docente para la promoción del gusto por la lectura literaria en base a la detección de: 1) elementos intradiégeticos relacionados de algún modo con lo escrito y la competencia literaria en su doble vertiente de recepción y producción de textos (personajes relacionados con la lectura y la escritura y objetos portadores de texto); 2) tipologías o géneros discursivos y géneros literarios (Bajtin, 1997); 3) interesantes referencias literarias concebidas como intertextualidades lectoras (Martínez Fernández, 2001; Mendoza, 2001), centrándonos especialmente en las referencias a diferentes tipos de obras clásicas de la literatura. Hemos preferido dejar a un lado las numerosas referencias culturales (históricas, sociales, científicas) así como los homenajes cinematográficos (concernientes, en su mayoría, al género western) que 
incluye este elaborado y complejo juego, algunos de los cuales han sido recientemente analizadas (Chun, 2020; Ronald y Reid, 2020).

\section{Aproximación al objeto de estudio: de las novelas de Estefanía al western interactivo}

En este caso, se trata de un juego en inglés con subtítulos y textos en castellano que explota al máximo, gracias a las opciones técnicas de las consolas de nueva generación, las posibilidades de indagación y descubrimiento del denominado género sandbox o de mundo abierto, en el que el jugador puede no seguir una determinada línea de acción narrativa y explorar por su cuenta el mundo de ficción virtual. Encontramos, respecto al modo historia del juego, una estructura narrativa de carácter literario organizada en seis largos capítulos (subdivididos a su vez en misiones, desde un mínimo de 6 a un máximo de 18), con un prólogo y un epílogo dividido en dos partes.

Como todo buen relato que pretende hacer épica de un momento histórico sometido a grandes cambios y unos personajes entrañables pero sobrepasados por las circunstancias, RDR2 constituye una historia crepuscular y de redención. La historia que el jugador deberá ir leyendo de manera interactiva retrata la decadencia progresiva de una notoria banda de forajidos, dirigida por Dutch Van der Linde, en los Estados Unidos de finales del siglo XIX. Sigue al protagonista, Arthur Morgan, cuyo temperamento violento y lealtad incondicional al líder de la banda lo convierten en el asesino a sueldo más confiable de la pandilla. La historia comienza después del fracaso del grupo en una misión de robo en la ciudad de Black Water. Toda la cuadrilla se encuentra huyendo constantemente de la ley, desesperada por iniciar de forma anónima un nuevo asentamiento. Posteriormente, la tropa se moverá por diferentes asentamientos debido a un evento inesperado tras otro. Los planes aparentemente ambiciosos, pero ambiguos y algo volubles, de Dutch para emancipar inadvertidamente a toda la banda de su vida criminal fracasan en el proceso, lo que lleva a la muerte innecesaria de algunos de los miembros. Mientras Dutch pierde el control de la realidad y es constantemente manipulado por un astuto secuaz, Micah Bell, Arthur 
sigue estando en el centro de la ejecución de los planes de Dutch hasta que le diagnostican tuberculosis terminal. Enfrentado así a su muerte inminente, acepta las malas acciones de su pasado y el liderazgo inestable de Dutch. Ante un dilema moral entre su lealtad a Dutch y salvar a sus amigos y a él mismo del atroz camino al que el jefe de la banda está llevando a todos, Arthur finalmente elige un camino altruista y de sacrificio personal para ayudar a su mejor amigo, John Marston, a escapar del desastre con su esposa e hijo y finalmente muere, tras el último enfrentamiento con Dutch y Micah.

Se trata, en realidad, de una precuela, del juego homónimo que en 2009 nos presentaba las aventuras del personaje que relegará a Arthur Morgan como protagonista al final de esta historia, John Marston, en el que nos encontramos con 60 horas de storytelling, más de mil personajes con diferentes líneas de diálogo, en un proceso de creación y desarrollo que ha durado casi una década. Un auténtico mundo de ficción interactivo (Planells, 2015) que permite una calidad de imagen en $4 \mathrm{~K}$, característica técnica que consiente un detallismo en elementos de ambientación como el paisaje o la recreación histórica de ciudades, favoreciendo lo que se conoce como experiencia inmersiva. Por su complejidad, combina diferentes categorías de clasificación propuestas en función de sus posibilidades narrativo-literarias (SernaRodrigo, 2016; Serna-Rodrigo, 2020), ya que se trata de una mezcla de aventura gráfica y narrativa digital no lineal influenciada por estructuras, temáticas y personajes propios de la literatura y el cine.

\section{1. Elementos intradiegéticos, géneros discursivos y usos sociales del escrito}

En este trabajo consideramos los siguientes elementos intradiegéticos (algunos de los cuales configuran posibilidades de lectura íntegra dentro del marco ficcional que se propone en el juego como contexto comunicativo) como categorías de análisis para el estudio del videojuego desde una perspectiva de fomento lector, en un caso de estudio que pretende considerar Red Dead Redemption 2 como una historia interactiva que va más allá de las limitaciones de otros 
géneros más habituales (como los meros shooter o los juegos tipo farming ambientados en el Salvaje Oeste como espacio mítico sin referencias históricas o literarias, con planteamientos de jugabilidad más simples).

\section{Tabla 1}

La lectura y la escritura en RDR2

\begin{tabular}{lc}
\hline Categoría & Tipos \\
\hline Personajes lectores & Principales, secundarios, \\
Personajes escritores & anecdóticos \\
Objetos portadores & Libros \\
& Estampas \\
& Mapas \\
& Carteles \\
& Comida \\
Géneros discursivos & Bebida \\
& Productos consumibles \\
& Diarios \\
& Cartas \\
Géneros literarios & Mapas \\
& Periódicos \\
& Recetas \\
& Notas \\
& Catálogos \\
\hline
\end{tabular}

Fuente: Elaboración propia

En primer lugar, es interesante observar cómo en un juego comercial, de ambientación western, se incluyen elementos más bien propios de desarrolladores indie, como es el caso de una cierta diversidad de personajes leyendo, que pueden interpretarse como modelos lectores dentro de la ficción que supone la narrativa interactiva del juego, construida como un espacio de lectura (Gutiérrez Ángel, 2020) y de aprendizaje socioemocional (Esnaola y Levis, 2008), sobre todo, a medida que avanza la historia (cabe destacar, en este sentido, la dramática evolución psicológica de los personajes principales). En el campamento de la banda de forajidos e inadaptados de la que forma 
parte Arthur Morgan, el protagonista, nos encontramos con personajes secundarios que leen diferentes tipos de libros: novelas de aventuras y otros géneros folletinescos propios de la época, novelas románticas, de aventuras, de terror, e incluso del Oeste, quienes además solicitan diferentes títulos al protagonista, que deberá encontrar los ejemplares para sus compañeros. Mención aparte merece el líder y cerebro de la banda: Ducth van Der Linde, lector competente, de firmes convicciones en cuanto a la desobediencia civil y la autosuficiencia como modos de vida legítimos en una época de cambios; es el personaje que ingenia y planifica las diferentes misiones (asaltos a trenes, robo de bancos, atracos a casas y diligencias, estrategias para despistar a las fuerzas de la ley y el orden, etc.), a quien podemos observar leyendo, preparando discursos y arengando a la cuadrilla en diferentes momentos. En las ciudades, sobre todo en Saint Denis (una ciudad inventada que pretende ser la análoga virtual de la New Orleans histórica), nos encontramos a personajes anónimos leyendo en los parques u hojeando el periódico en las esquinas. En mitad de la naturaleza, en nuestros numerosos viajes a lo largo y ancho del territorio americano virtual que compone el extenso mapa del juego (mapa de un territorio ficticio), se producirán, además, encuentros fortuitos con diferentes interlocutores que leen a la sombra de un árbol o a la orilla de un río. Cabe añadir que, en el epílogo de la historia, el hijo de John Marston, Jack, criado por los forajidos, aislado del mundo civilizado y la vida social, acabará convirtiéndose en lector voraz.

Aparecen también, en este peculiar videojuego, personajes principales que escriben y además hacen gala de ello. En primer lugar, tenemos al ya mencionado Dutch van der Linde, que escribe poemas, arengas y discursos para el grupo. Posee una máquina de escribir y se expresa en un tono eminentemente literario y reflexivo ("Nos persiguen sin descanso porque representamos todo lo que temen"), sugiriendo una determinada perspectiva utópica de la vida outsider, idealista, anárquico, carismático e ilustrado pero cuya integridad moral acaba resquebrajándose bajo las presiones del mundo moderno.

Otros personajes secundarios son: Lillian Powell, escritora borracha en uno de los salones de Saint Denis, que parece encarnar un 
determinado tipo de autor decadente decimonónico; Theodore Levin, un escritor ambulante que viaja investigando personajes que se consideran leyendas del Oeste en lo que supone un evidente juego metaficcional sobre el mundo crepuscular de 1899; tenemos también a la encantadora Mary-Beth, uno de los personajes femeninos que pertenecen a la banda, compuesta a modo de comuna (una auténtica gang), quien acabará convirtiéndose en escritora de novelas románticas bajo el pseudónimo de Leslie Dupont y quien, en el epílogo (ubicado temporalmente en 1907), le regala un libro a John Marston (The Lady of The Manor); Evelyn Miller, por otra parte, es un personaje que funciona como amalgama de reconocidos escritores y filósofos norteamericanos de la denominada nature writing, como Ralph Waldo Emerson, Henry David Thoreau o el escocés John Muir. Además, el personaje protagonista (Arthur Morgan y después John Marston) lleva un cuaderno a manera de diario en el que escribe y dibuja regularmente, cuyas páginas, repletas de garabatos y anotaciones, quedarán a disposición del jugadorlector.

Por otra parte, se incluyen una infinidad de objetos portadores de texto, como estampas de cigarrillos coleccionables (un total de 144 estampas repartidas en 12 colecciones temáticas: pistoleros famosos; artistas, escritores y poetas; paisajes de Norteamérica; personajes femeninos que destacan por su belleza y otras mujeres famosas; flora de Norteamérica; actores y actrices, fauna de Norteamérica; maravillas del transporte; deportistas, boxeadores y otros campeones del mundo; inventos del siglo XIX como el telégrafo, la pianola, la máquina de escribir, la cámara fotográfica o la silla eléctrica; razas de caballos; americanos famosos como personalidades políticas y militares), también mapas diversos, carteles, envoltorios de comida, bebida y otros productos consumibles (munición, medicinas, utensilios de caza y pesca, productos de higiene, ropa $\mathrm{y}$ calzado, objetos para el mantenimiento y cuidado de la montura, etc.), además de los diferentes catálogos e inventarios donde todos estos productos se encuentran disponibles, cuya lectura será inevitable en la mayoría de los casos para seguir avanzando en el juego. 
Asimismo, se incluyen en RDR2 una serie de géneros discursivos significativos. En cuanto a las posibilidades de fomento de la lectura, resulta interesante realizar un breve apunte sobre estos textos diversos. Como textos literarios legibles, aparecen, además de la referencia a otros géneros, tres novelas del Oeste. Como textos no literarios, legibles de manera íntegra o fragmentaria, se incluyen 42 periódicos, 73 cartas y misivas de diferente tipo, seis carteles de fugitivo, 28 recetas (remedios, mejunjes, tónicos), 15 notas, además de diversos libros de temática científica, social o de carácter práctico $(E l$ cultivo, el campo y la cetrería, Divagaciones en bosques y llanuras, etc.).

En cualquier caso, resulta admirable y altamente útil la inclusión de todos estos elementos que obligan a la lectura textual por parte del jugador. Es cierto que esta participación lectora en mitad de una narrativa de acción histórica, desde cierto enfoque funcional y comunicativo de la lectoescritura, en un marco ficcional (no solo instructivo), es una característica compartida por otros videojuegos, como los títulos de la saga Assassin's Creed, y ha sido estudiada en trabajos anteriores (Serna-Rodrigo, 2019), pero nos gustaría incidir en la pluralidad de opciones bien contextualizadas del título analizado aquí como caso ejemplar.

\section{2. Referencias literarias: intertextualidades a favor del fomento lector}

Como análisis previo al ejercicio de aplicación práctica, hemos indagado la presencia de referencias a obras clásicas de la literatura, en virtud del concepto de intertexto lector como conjunto activo de saberes, estrategias y recursos lingüísticos y pragmático-culturales que se actualizan en cada recepción literaria, con la finalidad de construir nuevos conocimientos significativos que pasan a integrarse en la competencia literaria (Mendoza, 2001). En este sentido, proponer un videojuego para trabajar la competencia literaria implica desarrollar en los alumnos criterios valorativos sobre la significación cultural y artística del texto encaminada hacia la recepción constructiva del 
significado de la narrativa interactiva como discurso literario que puede incluir, efectivamente, citas o referencias a otras obras.

Además de diferentes ecos bíblicos en los títulos de diversas misiones ("La consecuencia del Génesis", "El que esté libre de pecado", "Bienaventurados los pacificadores", “Sodoma? De vuelta a Gomorra", "Una nueva Jerusalén"), y de la referencia — a través de la inclusión de una horripilante horda de forajidos caníbales que viven en los bosques - a la novela Blood Meridian or The Evening Redness in the West, de Cormac McCarthy (1985), un neowestern épico de 1985, encontramos en RDR2 claras intertextualidades que nos remiten a diferentes tipos de clásicos literarios.

En primer lugar, existen referencias a clásicos decimonónicos anglosajones como Wuthering Heights, de Emily Brönte (1847). En el capítulo 3, aparecen Los Braithwaite y los Gray, dos familias enfrentadas que viven a lado y lado de la ciudad de Rhodes, equiparables a los Linton y los Earnshaw de Cumbres borrascosas, unos más incivilizados y los otros más refinados, cuyos respectivos descendientes acabarán trágicamente enamorados. Tanto el apellido Linton (Mary Linton) como el de Bronte dan nombre, además, a diferentes personajes del juego.

Como comentábamos anteriormente, aparece también la referencia a los clásicos estadounidenses de la escritura al aire libre y el amor por la vida salvaje (nature writing), considerados los padres de la ecocrítica, en el personaje secundario de Evelyn Miller, un personaje amalgama, "contemporáneo" de H. D. Thoreau, R. W. Emerson y John Muir, a quienes parafrasea en sus ideas sobre la naturaleza y el oficio de escritor.

Por otra parte, nos encontramos con la misión titulada "Sale, perseguido por un orgullo herido" ("Exit pursued by a bruised ego") en el capítulo 2, donde el personaje del veterano Hosea propone al jugador ir a cazar un oso a las montañas, una experiencia que resultará especialmente intensa, teniendo en cuenta además las excelentes características técnicas y visuales del juego. El título hace referencia a 
la acotación más famosa de Shakespeare en Winter's Tale (1623): “Exit pursued by a bear", presagiando la muerte fuera de escena de Antígona y planteando así un determinado horizonte de expectativas en la metanarrativa del juego (cabe añadir que en el capítulo 1 se incluye previamente la misión inicial "Entra, perseguido por un recuerdo" en el que debíamos rescatar a John Marston en las montañas nevadas, atacado por una manada de lobos).

En este breve repaso a las referencias a obras literarias de RDR2, resulta imprescindible mencionar otro tipo de clásicos a los que se evoca e incluso se menciona en el juego: los clásicos medievales y, más concretamente, la literatura artúrica. Existen diversas aproximaciones al estudio de los paralelismos entre la épica caballeresca y la épica del western como género cinematográfico (Pérez Rubio, 2002; Quesada, 2007; Humphreys, 2012). El hecho es que en RDR2 puede observarse una determinada narrativa de la hazaña en un mundo decadente (cambiante en valores y modos de vida) y además en el juego, a través del joven Jack Marston como lector de novelas de caballería, se realizarán diferentes referencias a la Materia de Bretaña (los caballeros de la Mesa Redonda, Lancelot, etc.) lo que permite relacionar la historia, desde un punto de vista de interpretación intertextual, con las obras de Geoffrey de Monmouth y Chrétien de Troyes.

Existen, asimismo, otras referencias a acontecimientos y personajes históricos, lo que permitiría el aprovechamiento didáctico de este videojuego para propuestas integradas de educación literaria y enseñanza de la historia: existen contenidos relativos a la Guerra Civil de Secesión; se trata en diversos capítulos la masacre de tribus indígenas americanas; se ridiculiza el $\mathrm{Ku}$ Klux Klan, se realiza una cierta crítica de la revolución industrial y sus efectos perjudiciales para el entorno en las grandes ciudades; aparecen más personajes análogos o amalgamas como Marko Dragic (Nikola Tesla), Dorothea Wicklow (activista del sufragio femenino), los Braithwaites y los Grays (que además de la referencia a la novela de Emily Bronte, pueden funcionar como posibles versiones ficcionales de los Hatfields y los McCoys, propietarios de mansiones y plantaciones esclavistas de tabaco y 
algodón); e incluso se referencia el accidente con una trampa para osos de Theodor Roosevelt, entre muchos otros.

\section{Funciones del profesor de literatura ante los videojuegos}

La educación tecnológica es clave en la formación profesional docente para adquirir competencias digitales, en un sentido crítico y lúdico a un mismo tiempo, que deben ser fortalecidas desde la formación del profesorado. Desde los inicios del siglo XXI se ha incrementado la necesidad de incluir las competencias digitales en las prácticas docentes $\mathrm{y}$, entre ellas, experiencias con mediación de videojuegos como materiales didácticos interactivos y propensos a la multimodalidad convergente de diferentes discursos estéticos (Gee, 2003; Ramírez, 2012; Torres-Toukoumidis et al, 2016; Correa, Duarte y Guzmán, 2017; Esnaola y Ansó, 2019; Ferragut y García, 2019; Martínez Urbano, 2021).

A partir de las funciones del profesor de literatura en sus distintos roles, descritas por Ballester (2015, p. 41), nos gustaría destacar, especialmente, aquellas que - como motivador, organizador, evaluador, modelo, transmisor y gestor - consideramos que podrían ser especialmente relevantes respecto al uso de los videojuegos para la educación literaria: motivar, estimular y orientar el aprendizaje y las capacidades del estudiante; intermediar entre la información de todo tipo y el proceso de construcción del conocimiento; adoptar una óptica interdisciplinaria y global de la educación; ser usuario de las diferentes tipologías literarias y textuales; mantener actitudes positivas hacia el uso y la integración de las TIC; ser un profundo modelo lector y, por lo tanto, fomentar la lectura; transmitir no solo conocimientos, sino actitudes sociales y culturales; seleccionar textos adecuados desde el interés y el gusto del estudiante y que, a su vez, tengan en cuenta las exigencias del currículum vigente.

Como investigador e innovador, el docente ha de reflexionar sobre el proceso de enseñanza-aprendizaje; conocer la diversidad metodológica; utilizar una metodología activa, participativa y colaborativa; conocer y practicar la formación de las TIC como recursos 
didácticos; actualizar los conocimientos tanto didácticos como comunicativos; analizar la propia práctica docente; provocar interrogantes y sistematizar las experiencias educativas; y practicar la investigación y reflexión críticas del propio proceso educativo. Llegados a este punto, también nos gustaría añadir que, en primer lugar, en su papel de investigador e innovador, el docente debe reconocer referencias lectoras y promover enlaces intertextuales en cualquier género y medio; y como consecuencia, ser usuario también de videojuegos desde la perspectiva del mediador $\mathrm{y}$, en definitiva, de gamer con criterios pedagógicos y como experto en didácticas específicas. En este sentido, la figura docente asume una serie de responsabilidades como intermediario respecto al fomento del gusto por la lectura de las obras consideradas clásicas desde una concepción multimodal de la lectura literaria (Caro Valverde, 2019).

Esta perspectiva afecta a los paradigmas de investigación en didáctica de la literatura y la lengua, lo que nos llevaría a plantear una revisión de los videojuegos no solo para la promoción del fomento y placer lector a partir de la experiencia de juego orientada por el docente, sino también para la inclusión de la interactividad multimedia en aprendizajes linguiísticos y literarios planificados teniendo en cuenta metodologías activas (por ejemplo, proyectos integrados); como experiencia cultural previa para las tareas de escritura, reescritura y traducción de textos en talleres de creatividad literaria y, en definitiva, para la integración de este medio en la planificación de secuencias didácticas.

\section{Conclusiones}

La lectura en pantalla interactiva, inserida en un marco ficcional (donde los textos tienen una determinada finalidad comunicativa y exigen la participación lectora del jugador mediante la aplicación de ciertos usos sociales-virtuales del escrito) nos lleva a la conclusión de un acto lector eminentemente lúdico que forma parte de las prácticas de ocio digital pero que, como en el caso que presentamos, no menoscaba la inclusión de múltiples referencias intertextuales a clásicos literarios. 
La finalidad de la lectura se ludifica y se narrativiza, obligando a redefinir los procedimientos y propuestas de fomento lector y las funciones del profesor de literatura, que ha de recorrer nuevos itinerarios para acceder y permitir el acceso a los referentes culturales y al conocimiento de la tradición literaria a través de nuevas estrategias de mediación, a partir de la experiencia de juego. Y como apunta Martínez Urbano (2021, p. 330): “Aprender a leer videojuegos es una forma de reforzar la formación literaria desde el siglo XXI y uno de los caminos que los docentes hemos de seguir para lograr que nuestros alumnos puedan acceder a la cultura".

En este sentido, Serna-Rodrigo y Rovira-Collado (2016), reflexionando sobre videojuegos que toman referencias de obras clásicas y mundos literarios, como Dante's Inferno, inspirado en la Divina Comedia, apuntaban:

\begin{abstract}
Esta clase de videojuegos, además de enriquecer la competencia lectoliteraria de sus usuarios, pueden llegar a despertar el interés de estos en las obras clásicas pues, si disfrutan con el juego, es posible que el libro ya no sea un total desconocido para ellos, sino un modo de aprender más sobre esa historia que les ha atrapado. Los videojuegos conforman, por tanto, una parte significativa del universo transmedia de los lectores más jóvenes, ya que son parte de su cultura, sus referencias y su tiempo de ocio (p. 781).
\end{abstract}

Es cierto que el caso de RDR2 puede suponer, por su complejidad técnica y narrativa, un caso aislado. Pero consideramos que marca muy bien el camino hacia lo que pueden ofrecer los videojuegos en contextos de investigación educativa. Aquí simplemente hemos pretendido exponer cómo aprovechar el game play como experiencia de lectura llevada a cabo por el docente en su función de investigador e innovador, apostando por el fomento de la lectura de los clásicos en un modelo ecléctico, multimodal y transmedia de lo que consideramos la educación literaria en el siglo XXI.

Hay que recordar, sin embargo, que autores como Italo Calvino (1993) ya contemplaban esta incursión de los clásicos como libros que ejercen una influencia particular, ya sea cuando se imponen por 
inolvidables, ya sea cuando se esconden en los vericuetos de la memoria mezclándose con el inconsciente colectivo o individual y convirtiéndose, así, en referentes culturales. En este sentido, uno de los objetivos de la investigación en didáctica de la literatura sería reconocer la genealogía de algunos clásicos cuyos personajes, temáticas o argumentos, de manera fragmentaria o no, se incluyen en narrativas interactivas como los videojuegos.

La investigación en didáctica de la literatura y la lengua ha de considerar la inclusión de otros medios, como el cómic y los videojuegos, en virtud de la multimodalidad y la interactividad lúdica de los procesos de aprendizaje y teniendo en cuenta lo que conlleva el desarrollo de la competencia mediática y digital (Pérez Rodríguez, 2020a y 2020b). Los clásicos se reescriben constantemente a través de estos géneros y medios, y ante el docente aparece el reto de conocer estos nuevos senderos para la lectura literaria, de recorrerlos didácticamente y llevarlos a las aulas mediante propuestas que los incluyan como experiencias culturales a comentar, el reto de analizarlos y compartirlos (como hemos pretendido sucintamente en este trabajo), porque forman parte de las prácticas letradas y de ocio digital de niños y adolescentes.

Por otro lado, en lo referente a la lectura de los clásicos literarios, un conocimiento de mera antología no basta (Ordine, 2017, p. 17), resulta necesario realizar un esfuerzo por mostrar la interacción significativa de las obras con las nuevas tecnologías y con las nuevas maneras de contar historias, con los demás saberes y con la vida misma. Sin embargo, es cierto que los clásicos están ahí siempre, irreductibles, adaptados, actualizados, multimodales, interactivos, en ocasiones escondidos tras veladas referencias intertextuales. Solo hay que saber leer de manera crítica.

\section{Referencias bibliográficas}

Baker, N. A. (2013). Extra lives: why video games matter. Choice, 48 (7), 1279-1285. 
Ballester, J. (2015). La formación lectora y literaria. Barcelona: Graó.

Ballester-Roca, J., e Ibarra-Rius, N. (2019). Autobiografías lectoras y clásicos en la educación literaria de los futuros docentes e investigadores. Tejuelo: Didáctica de la Lengua y la Literatura, 29, 3166. doi: https://doi.org/10.17398/1988-8430.29.31.

Bajtin, M. (1997). Estética de la creación verbal. México: Siglo XXI.

Calvino, I. (1993). Por qué leer los clásicos. Barcelona: Tusquets.

Caro Valverde, M. T. (2019). El clásico, irreductible. Innovación didáctica de la creación literaria multimodal. Tejuelo: Didáctica de la Lengua y la Literatura, 29, 245-274. doi: 10.17398/1988-8430.29.245.

Cerrillo, P., y García Padrino, J. (coords.) (1996). Hábitos lectores y animación a la lectura. Cuenca: Universidad de Castilla-La Mancha.

Chun, B.S. (2020). Narrative Structure and Ludonarrative Dissonance in the Video Game Red Dead Redemption 2. Journal of the Korea Entertainment Industry Association, 14 (5), 59-72.

Castro, A. de (2015) Maximum Consequentia: un videojuego educativo para ejercitar la comprensión lectora. Innovación Educativa, 25, 191-206. doi:10.15304/ie.25.1919.

Colomer, T. (1995). La adquisición de la competencia literaria. Textos de Didáctica de la Lengua y la Literatura, 4, 8-22.

Colomer, T. (2010). La evolución de la enseñanza literaria. Biblioteca Virtual Miquel de Cervantes. Recuperado de: http://www.cervantesvirtual.com/obra/la-evolucion-de-la-ensenanzaliteraria/.

Cope, B. y Kalantzis, M. (2009). Multiliteracies: New literacies, new learning. Pedagogies: An International Journal, 4 (3), 164-195.

Cope, B. y Kalantzis, M. (2015). The things you do to know: An introduction to the pedagogy of multiliteracies. En B. Cope y M. Kalantzis (eds.), A pedagogy of multiliteracies: Learning by design (pp. 1-36). London: Palgrave. 
Correa, R. I.; Duarte, A., y Guzmán M. D. (2017). Horizontes educativos de los videojuegos: propuestas y reflexiones de futuros maestros y educadores sociales. Educar, 53 (1), 67-88. doi: https://doi.org/10.5565/rev/educar.849.

Esnaola, G. A., y Levis, D. (2008). La narrativa en los videojuegos: un espacio cultural de aprendizaje socioemocional. Revista Electrónica Teoría de la Educación, 9 (3), 48-68. doi: https://doi.org/10.14201/eks.16789.

Esnaola, G., y Ansó, M. B. (2019). Competencias digitales lúdicas y enseñanza. REIDOCREA, 8, 399-410. Recuperado de: https://www.ugr.es/ reidocrea/8-31.pdf.

Ferragut, D., y A. García (eds.) (2019). Ensayos y errores. Arte, ciencia y filosofía en los videojuegos (pp. 29-48). AnaitGames.

Gee, J. P. (2003). What videogames have to teach us about learning and literacy. Nueva York: MacMillan.

Gutiérrez Ángel, N. (2020). El videojuego y la lectura literaria: nuevo espacio para los nativos digitales. Contextos Educativos, 25, 145159. doi: $10.18172 /$ con.4250.

Hernández Ortega, J., y Rovira-Collado, J. (2020). Diseño de proyectos transmedia para la Educación Literaria en el aula de Educación Secundaria. RESED. Revista de Estudios Socioeducativos, 8, 80-94.

doi:

http://dx.doi.org/10.25267/Rev_estud_socioeducativos.2020.i8.7.

Herrera Pérez, C. A. y Saavedra Rey, A. S. (2012). La formación de lectores de literatura capaces de alcanzar el efecto estético por medio de la intertextualidad. Nodos y nudos: revista de la Red de Calificación de educadores, 4 (33), 38-48. doi: 10.17227/01224328.2072.

Humphreys, S. (2012). Rejuvenating "Eternal Inequality" on the Digital Frontiers of Red Dead Redemption. Western American Literature, 47 (2), 200-215. doi: 10.1353/wal.2012.0048.

Jenkins, H. (2006). Convergence Culture: Where Old and New Media Collide. Nova York: NY University Press.

López, A., Jerez, I., y Encabo, E. (2015). Sobre nuevas alfabetizaciones lectoras. Las experiencias mediáticas integradas y los lectores navegantes. En Santiago Yubero y Elisa Larrañaga (Coords.) Propuestas socioeducativas para la alfabetización lectora. UCLM. 
Martínez Fernández, J. E. (2001). La intertextualidad literaria. Madrid: Cátedra.

Martínez Urbano, P. (2021) El lenguaje videolúdico y la educación literaria. Los videojuegos en la formación del lector competente. Tesis Doctoral. Universitat de València.

Mayoral, J. A. (ed.) (1987). Estética de la recepción. Madrid: Arco Libros.

Méndez, J. (2017). Les adaptacions de clàssics literaris en la formació de mestres: la narrativa breu medieval. Lenguaje y Textos, 45, 71-88. doi: https://doi.org/10.4995/lyt.2017.7643.

Mendoza, A. (2001) El intertexto lector. El espacio de encuentro de las aportaciones del texto con las del lector. Cuenca: Universidad de Castilla-La Mancha.

Mendoza, A. (2004). La educación literaria: bases para la formación de la competencia lecto-literaria. Málaga: Ediciones Aljibe.

Ordine, N. (2017) Clásicos para la vida. Una pequeña biblioteca ideal. Barcelona: Acantilado.

Pérez Rodríguez, M. A. (2020a). Enseñar la competencia mediática. La alfabetización en la era transmedia. En J. Ballester y N. Ibarra (coords.) Entre la lectura, la escritura y la educación. Paradigmas de investigación en Didáctica de la Literatura y la Lengua (pp. 121-138). Madrid: Narcea.

Pérez Rodríguez, M. A. (2020b). Homo sapiens, homo videns, homo fabulators. La competencia mediática en los relatos del universo transmedia, ICONO14. Revista Científica de Comunicación y Tecnologías emergentes, 18 (2), 16-34. doi: https://doi.org/10.7195/ri14.v18i2.1523.

Pérez Rubio, P. (2002 [1995]). El western, viaje iniciático por los mapas de la épica. Biblioteca virtual Miguel de Cervantes. Recuperado de: http://www.cervantesvirtual.com/nd/ark:/59851/bmcbg2n5.

Planells, A. J. (2015). Videojuegos y mundos de ficción: de Super Mario a Portal. Madrid: Cátedra.

Quesada Navidad, R. (2007). La evolución del género western en el cine norteamericano: de la modernidad a la postmodernidad. Tesis doctoral. Universidad de Jaén. 
Ramírez, A. (2012). Posibilidades educativas de los videojuegos y juegos digitales en educación secundaria obligatoria. En V. Marín (coord.), Los videojuegos y los juegos digitales como materiales educativos (pp. 133-174). Madrid: Síntesis.

Ronald, I. y Reid, A. (2020). The Wild West: Accuracy, Authenticity and Gameplay in Red Dead Redemption 2. Media Education Journal, 66, 15-23. Recuperado de: https://rke.abertay.ac.uk/ws/portalfiles/portal/17590756/Donald_Reid_ TheWildWest_Published_2020.pdf.

Rovira-Collado, J. (2019). Clásicos literarios en constelaciones multimodales. Análisis de propuestas de docentes en formación. Tejuelo: Didáctica de la Lengua y la Literatura. Educación, 29, 275312. doi: 10.17398/1988-8430.29.275.

Scolari, C. A. (2013). Narrativas transmedia. Cuando todos los medios cuentan. Barcelona: Deusto.

Scolari, C. (2018). Narrativas transmedia, nuevos alfabetismos y prácticas de creación textual. Conflictos y tensiones en la nueva ecología de la comunicación. Lectoescritura digital (pp. 45-52). Ministerio de Educación y Formación Profesional.

Senís, J. y Cerrillo, P. C. (2005). Nuevos tiempos, ¿nuevos lectores? Ocnos: revista de estudios sobre lectura, 1, 19-34.

Serna-Rodrigo, R. (2019). La participación lectora en la narrativa del videojuego. e-SEDLL, 1, 181-196. Recuperado de: https://cvc.cervantes.es/literatura/esedll/pdf/01/12.pdf.

Serna-Rodrigo, R. (2020). Posibilidades de los videojuegos en el ámbito de la Didáctica de la Lengua y la Literatura. Una propuesta de clasificación. EDMETIC, Revista de Educación Mediática y TIC, 9(1), 104-125. doi: 10.21071/edmetic.v9i1.12245.

Serna-Rodrigo, R. y Rovira-Collado, J. (2016). Aportaciones de los videojuegos a la Educación Literaria. En M. T. Tortosa, S. Grau, y J. D. Alvárez (coords.) XIV Jornadas de Redes de Investigación en Docencia Universitaria. Investigación, innovación y enseñanza universitaria: enfoques pluridisciplinares (pp. 772-786). Alicante: Universitat d'Alacant.

Sotomayor, M. V. (2013). ¿Qué hacemos con los clásicos? Algunas reflexiones para los futuros docentes. Lenguaje y Textos, 38, 29-35. 
Torres-Toukoumidis, A.; Romero-Rodríguez, L.; PérezRodríguez, M. A., y Björk, S. (2016). Desarrollo de habilidades de lectura a través de los videojuegos: Estado del arte. Ocnos, 15 (2), 37 49. doi: https://doi.org/10.18239/ocnos_2016.15.2.1124. 
Tejuelo, no 34 (2021), págs. 195-220. Los clásicos como resistencia: la lectura literaria en el marco ... 